\title{
THE STRASBOURG CONGRESS
}

Comptes Rendus du Congrès international des Mathématiciens (Strasbourg, 1920). Publiés par Henri Villat. Toulouse, Édouard Privat, 1921. xlvii $+670 \mathrm{pp}$.

The Strasbourg Congress is particularly interesting as the first congress after the War, and its proceedings indicate the results of researches made during the War as well as forecast those to be made later. If the reader will glance at the list of topics in the volume, he will notice that many of those topics have continued to develop since the time of the Congress, and that some of them (for instance that of general complex numbers) have since then reached a certain definitive stage.

The mathematical part of the volume before us consists of five general lectures, by Messrs. Larmor, Dickson, de la Vallée-Poussin, Volterra, and Nörlund, and of 68 other papers and résumés of papers. Among the latter, 32 are in arithmetic, algebra and analysis, 10 are in mathematical physics and applied mathematics, and 9 are on questions philosophical, historical, and pedagogical. An adequate review of the volume would necessitate an analysis of each of these general lectures and papers; because of lack of space we give merely abstracts of the general lectures.

Larmor: Questions in physical interdetermination.

Sir Joseph Larmor's lecture is a masterly critical discussion of modern theories of spacetime, with particular reference to the two questions of physical interdetermination and of the postulation of a Hamiltonian principle of extremal variation of action.

There is complete statical interdetermination in a freely jointed frame of rigid bars without redundant members. Another instance of complete statical interdetermination is given by the newtonian potential, which is determined throughout free space or so long as no attracting matter is traversed, by its values in any region. This latter interdetermination holds for any isotropic uniform elastic medium, whether material solid or ether. Complete static interdetermination can take place only in real spaces; freedom demands that at least one dimension shall be imaginary. Radiation is the principal, if not the sole method of production of change of physical systems out of direct contact. If radiation it to take place in waves and if the medium is isotropic, time restricted to a single dimension, it can be shown that space must have three dimensions. The variation of the total intrinsic action of a physical system is postulated to be extremal. The space is thus considered to exist apart from the physical processes that take place in it. In special relativity, then, a space is characterized by its potentials. The moving 
sources or masses are attached to a framework, whose displacement presents an extremal variation of the action. The tensor thus introduced gives, by integration over a barrier surface, the mechanical effects on each source or mass. The action involves deformation of the space itself rather than disturbance of the medium, as in the ether theory. The principle of a space distribution of an action function, whose integral is postulated to be of extremal variation (centers of activity being cut out by barrier surfaces), is the only known system assigned by local characteristics which permits the deduction of relations between distant parts of the medium. This system is at variance with the Einstein prediction of displacement of the lines of the spectrum. The system also leads to a complete self-consistent theory before all atoms of the same substance are identified. A test of the validity of the theory is thus the existence of identical atoms, whose existence can be detected by radiation to a distance.

Dickson: Some relations between the theory of numbers and other branches of mathematics.

The methods of one branch of mathematics are a frequent aid in the development of another branch. Such theories as are developed by the use of methods and by analogy with the results of a different field are then of particular interest. As an illustration, Dickson shows how geometric methods may be used to find the rational solutions of certain Diophantine equations. Dickson then presents a new method, involving algebraic numbers and integral quaternions, for finding formulas for the integral solutions of Diophantine equations while the parameters take only integral values. The remainder of the lecture is devoted to an illustration of the nature of modular invariants, as developed by Dickson and his pupils, and to the extension of an application by Mordell of the theory of algebraic invariants.

De la Vallée-Poussin: Sur ies fonctions à variation bornée.

Modern work in real variables may be divided roughly into two classes, the first the theory of linear partial differential equations and the second the theory developed in the present century by Baire, Borel, Lebesgue, and their followers. The theories are intimately connected in various ways; for example, by the theory of the development of arbitrary functions in terms of solutions of ordinary differential equations. The purpose of the entire lecture is to point out the central position occupied by functions of bounded variation in this second class of topics on real variables. Thus every indefinite integral in the sense of Lebesgue is a function of bounded variation. If an integrable function $f(x)$ is defined in an interval $a \leqq x \leqq b$, the function

$$
F(E)=\int_{E} f(x) d x
$$


is an example of a fonction d'ensemble, where $E$ is any measurable point set (ensemble) contained in the interval; the function $F(E)$ has also the additive property, namely that

$$
F\left(E_{1}\right)+F\left(E_{2}\right)+\cdots+F\left(E_{n}\right)+\cdots=F(E),
$$

if $E$ is the sum of the non-overlapping sets $E_{1}, E_{2}, \ldots, E_{n}, \ldots$ The fonctions d'ensemble are of the highest importance in the study of measure and of integration. It can be shown that every additive fonction d'ensemble is also a point function of bounded variation and conversely.* It seems probable that in future developments functions of bounded variation are destined to play a central role, not merely as a class of functions to be developed in terms of given sets of functions, but also in connection with the theory of the differentiation of functionals.

\section{Volterra: Sur l'enseignement de la physique mathématique.}

Senator Volterra, as one who has not only spent a lifetime in teaching mathematical physics but has made many diverse and fundamental contributions to the field, is eminently fitted to discuss methods of presentation of the general subject. Volterra commences his lecture by outlining the history of his subject. The analytical physics to be considered is not that developed in the heroic age, up to the time of Lagrange, nor is it the most recent age which has just begun, of the great modern work in relativity and the constitution of matter. The theory of the most importance at present and yet that has reached a certain degree of finality in its methods and results is that due to Lagrange, Laplace, Green, Fourier, Cauchy, Maxwell, Riemann, in short, the applications to physics of partial differential equations. The model of exposition of a physical theory is Lagrange's masterpiece, where the writer commences with a historical introduction, arrives at a mathematical formulation of his problems, and finally proceeds with the solution of the purely mathematical problems. This plan is followed directly by Volterra. The unification of the theory of anaytical physics is to be secured by means of partial differential equations, and that this can be done is made clear by the first part of the course which Volterra suggests, the derivation of the partial differential equations and the formulation of the precise mathematical problems involved. The second part of the course naturally consists in the classification of differential equations according to their characteristics, and the solution of the boundary value problems which arise. These problems my be solved by the method of Green (fundamental solutions), that of characteristics, or that of

* In this connection and elsewhere de la Vallée-Poussin refers to his memoir of 1915 in the American Journal, a reference which should read to the 1915 volume of the Transactions of THIs Society. 
[October,

simple solutions as in Fourier's series. The third and last part of the course is the treatment of many problems which could not be solved or could be only partially solved by the former methods, and of some additional problems. These problems are unified by the notion of functions of lines, and lead us to integral equations, integro-differential equations, equations in functional derivatives, etc. The course thus outlined by Volterra has the advantage of unifying and systematizing analytical physics, and also of economy and of separating distinctly the physical notions and the mathematical processes. Every reader will hope that Volterra will be able actually to prepare and publish the course he suggests, not only a work of science but also a work of art.

Nörlund: Sur les équations aux différences finies.

This lecture is an exposition of some of the author's striking results on difference equations. In the equation

$$
F(x+1)-F(x)=\varphi(x),
$$

$\varphi(x)$ is a given function of the complex variable $x$, where $F(x)$ is to be determined. Equation (1) has been studied by various writers, including Euler, Plana, Abel, and Cauchy. Guichard showed that, if $\varphi(x)$ is an entire function, then (1) always has as a solution an entire function which can be expressed as a definite integral. Appell likewise proved this result, obtaining the solution as an infinite series. The solution of (1) is not uniquely defined, even when restricted to be an entire function. For to a solution may be added any function of period unity, and the sum will be an entire function, a solution of (1). Nörlund introduces the notion of principal solution, and studies its properties by means of divergent series.

The form and typography of the volume are excellent throughout, for the volume is uniform with and by the same publishers as the annales de la Falculté des Sciences de Toulouse.

J. L. WALSH 